\title{
ON BEST PROXIMITY PAIR THEOREMS AND FIXED-POINT THEOREMS
}

\author{
P. S. SRINIVASAN AND P. VEERAMANI
}

Received 27 November 2001

The significance of fixed-point theory stems from the fact that it furnishes a unified approach and constitutes an important tool in solving equations which are not necessarily linear. On the other hand, if the fixed-point equation $T x=x$ does not possess a solution, it is contemplated to resolve a problem of finding an element $x$ such that $x$ is in proximity to $T x$ in some sense. Best proximity pair theorems analyze the conditions under which the optimization problem, namely $\min _{x \in A} d(x, T x)$ has a solution. In this paper, we discuss the difference between best approximation theorems and best proximity pair theorems. We also discuss an application of a best proximity pair theorem to the theory of games.

\section{Introduction}

Many problems of practical interest are formulated as an operator equation $F x=0$ where the operator $F$ is defined on some suitable space. Often this operator equation is solved by recasting it as a fixed-point equation $T x=x$ such that the solution to the latter will yield a solution to the corresponding operator equation $F x=0$. For instance, existence of fixed-point to the equation $T x=F x+x$, whenever $F x+x$ is meaningful, is precisely a solution to the operator equation $F x=0$. The significance of this unified approach is that it serves as an important tool in solving linear as well as nonlinear equations. Further, fixedpoint theory has gained impetus, due to its wide range of applicability, to resolve diverse problems emanating from the theory of nonlinear differential equations, theory of nonlinear integral equations, game theory, mathematical economics, control theory, and so forth.

On the other side of the spectrum, if the fixed-point equation $T x=x$ does not possess a solution, then the next question that arises naturally is whether it is possible to find an element $x$ in a suitable space such that $x$ is close to $T x$ 
in some sense. Best approximation theorems provide affirmative answers to this poser. In fact, if $E$ is a normed linear space and if $T$ is a mapping with domain $K \subset E$, then a best approximation theorem furnishes sufficient conditions that ascertain the existence of an element $x_{0}$, known as best approximant, such that

$$
d\left(x_{0}, T x_{0}\right)=d\left(T x_{0}, K\right)
$$

where

$$
d(A, B)=\inf \{\|x-y\|: x \in A \text { and } y \in B\}
$$

for any nonempty subsets $A$ and $B$ of $E$. Indeed, a classical best approximation theorem, due to Fan [5], states that if $K$ is a nonempty compact convex subset of a Hausdorff locally convex topological vector space $E$ with a continuous seminorm $p$ and $T: K \rightarrow E$ is a single-valued continuous map, then there exists an element $x_{0} \in K$ such that

$$
p\left(x_{0}-T x_{0}\right)=d\left(T x_{0}, K\right)
$$

Later, this result has been generalized, by Reich $[11,12]$ and Sehgal and Singh [16], to the one for continuous multifunctions. It is remarked that Sehgal and Singh have also proved the following generalization [17] of the result due to Prolla [10].

If $K$ is a nonempty approximately compact convex subset of a normed linear space $X, T: K \rightarrow X$ is a multivalued continuous map with $T(K)$ relatively compact and $g: K \rightarrow K$ an affine, continuous, and surjective single-valued map such that $g^{-1}$ sends compact subsets of $K$ onto compact sets, then there exists an element $x_{0}$ in $K$ such that

$$
d\left(g x_{0}, T x_{0}\right)=d\left(T x_{0}, K\right)
$$

In the setting of Hausdorff locally convex topological vector spaces, Vetrivel, Veeramani, and Bhattacharyya [22] have established existential theorems that guarantee the existence of a best approximant for continuous Kakutani factorizable multifunctions which unify and generalize the known results on best approximations.

The following simple example shows that the requirement of continuity assumption of the involved multifunction in Sehgal and Singh's result [16] cannot be relaxed. 
Example 1.1. Let $X=\mathbb{R}, K=[0,1], T: K \rightarrow 2^{X}$, and $g=I$, the identity map. Let $T: K \rightarrow 2^{X}$ be defined as follows:

$$
T(x)= \begin{cases}\{3\} & \text { if } x=0 \\ {[2,4]} & \text { otherwise }\end{cases}
$$

Then $T$ is upper semicontinuous but not lower semicontinuous. Also, it is clear that there is no $x \in K$ such that

$$
d(x, T x)=d(T x, K)
$$

Although a best approximation theorem guarantees the existence of an approximate solution, it is contemplated to solve the problem of finding an approximate solution which is optimal. Best proximity pair theorems are pertinent to be explored in this direction. Indeed, if $T: A \rightarrow B$ is a multivalued mapping, a best proximity pair theorem provides sufficient conditions that ensures the existence of an element $x_{0} \in A$ such that

$$
d\left(x_{0}, T x_{0}\right)=d(A, B)
$$

The pair $\left(x_{0}, T x_{0}\right)$ is called a best proximity pair of $T$. Moreover, if the mapping under consideration is a self-mapping, it may be noted that under suitable conditions, this best proximity theorem boils down to a fixed-point theorem. Thus, best proximity pair theorems also serve as a generalization of fixed-point theorems.

Best proximity pair theorem (see [14]) analyzes the conditions under which the problem of minimizing the real-valued function $x \rightarrow d(x, T x)$ has a solution. It is evident that

$$
d(x, T x) \geq d(A, B) \quad \forall x \in A .
$$

Therefore, a nice solution to the above optimization problem will be one for which the value $d(A, B)$ is attained. In view of this standpoint, best proximity pair theorems are considered in this paper to expound the conditions that will ensure the existence of an element $x_{0} \in A$ such that

$$
d\left(x_{0}, T x_{0}\right)=d(A, B)
$$

The pair $\left(x_{0}, T x_{0}\right)$ is called a best proximity pair of the multifunction $T$. It may be noted that, since

$$
d(x, T x) \geq d(T x, A) \geq d(A, B) \quad \forall x \in A,
$$

an element satisfying the conclusion of a best proximity pair theorem is a best approximant $x_{0}$ but the refinement of the closeness between $x_{0}$ and its image $T x_{0}$ is demanded. Also, best proximity pair theorem sheds light in another direction, 
that is, it evolves as a generalization of the problem, considered by Beer and Pai [2], Sahney and Singh [15], Singer [18], and Xu [23], of exploring the sufficient conditions for the nonemptiness of the set

$$
\operatorname{Prox}(A, B):=\{(a, b) \in A \times B:\|(a-b)\|=d(A, B)\}
$$

The elements of $\operatorname{Prox}(A, B)$ are called proximal points of the pair comprising $A$ and $B$. In addition to the investigation of sufficiency for the nonemptiness of the set $\operatorname{Prox}(A, B)$, Pai $[8,9]$ and $\mathrm{Xu}[23]$ have expounded the uniqueness and characterization of proximal points.

\section{Preliminaries}

This section covers the preliminary notions and the results that will be required in the sequel to establish the main theorems.

Let $X$ and $Y$ be nonempty sets. The collection of all nonempty subsets of $X$ is denoted by $2^{X}$.

A multifunction or set-valued function from $X$ to $Y$ is defined to be a function that assigns to each element of $X$ a nonempty subset of $Y$.

If $T$ is a multifunction from $X$ to $Y$, then it is designated as $T: X \rightarrow 2^{Y}$, and for every $x \in X, T x$ is called a value of $T$.

For $B \subseteq Y$, the preimage or inverse image of $B$ under $T$, denoted by $T^{-1}(B)$, is defined as

$$
T^{-1}(B):=\{x \in X: T x \cap B \neq \phi\} .
$$

In what follows, it will be assumed that $X$ and $Y$ are topological spaces.

A multifunction $T: X \rightarrow 2^{Y}$ is said to be upper semicontinuous if for every closed subset $C$ of $Y$, its inverse image $T^{-1}(C)$ is closed in $X$.

It is known that if $T: X \rightarrow 2^{Y}$ is an upper semicontinuous multifunction with compact values, then $T(K)$ is compact in $Y$ whenever $K$ is a compact subset of $X$.

A multifunction $T: X \rightarrow 2^{Y}$ is said to be a compact multifunction if $T(X)$ is contained in a compact subset of $Y$.

The following result characterizes the upper semicontinuity of multifunctions.

Theorem 2.1 [1]. Let $X$ be a topological space and $Y$ a compact, Hausdorff topological space. A multifunction $T: X \rightarrow 2^{Y}$ is upper semicontinuous if and only if for every net $\left\{x_{\alpha}\right\}$ in $X$ and every net $\left\{y_{\alpha}\right\}$ in $Y$, the conditions $x_{\alpha} \rightarrow x, y_{\alpha} \in T x_{\alpha}$, and $y_{\alpha} \rightarrow y$ imply that $y$ is a member of Tx.

A characterization for lower semicontinuity is furnished below.

Theorem 2.2 [1]. Let $X$ and $Y$ be topological spaces. A multifunction $T: X \rightarrow 2^{Y}$ is lower semicontinuous if and only if for every net $\left\{x_{\alpha}\right\}$ in $X$ with $x_{\alpha} \rightarrow x$ and 
$y \in T x$, there is a subnet $\left\{x_{\beta}\right\}$ of $\left\{x_{\alpha}\right\}$ and a net $\left\{y_{\beta}\right\}$ such that $y_{\beta} \in T x_{\beta}$ and $y_{\beta} \rightarrow y$.

A multifunction $T: X \rightarrow 2^{Y}$ from a topological space $X$ to another topological space $Y$ is said to be a Kakutani multifunction [6] if the following conditions are satisfied:

(a) $T$ is upper semicontinuous;

(b) either $T x$ is a singleton for each $x \in X$ (in which case $Y$ is required to be a Hausdorff topological vector space) or for each $x \in X, T x$ is a nonempty, compact and convex subset of $Y$ (in which case $Y$ is required to be a convex subset of a Hausdorff topological vector space).

The collection of all Kakutani multifunctions from $X$ to $Y$ is denoted by $\mathscr{K}(X, Y)$.

A multifunction $T: X \rightarrow 2^{Y}$, from a topological space $X$ to another topological space $Y$, is said to be a Kakutani factorizable multifunction [6] if it can be expressed as a composition of finitely many Kakutani multifunctions.

The collection of all Kakutani factorizable multifunctions from $X$ to $Y$ is denoted by $\mathscr{K}_{C}(X, Y)$.

If $T=T_{1} T_{2} \cdots T_{n}$ is a Kakutani factorizable multifunction, then the functions $T_{1}, T_{2}, \ldots, T_{n}$ are known as the factors of $T$.

It may be noted that a Kakutani factorizable multifunction need not be convex valued even though each of its factors is convex valued.

\section{Best proximity pair theorems}

The following notions will be used in the sequel.

A multifunction $T: X \rightarrow 2^{Y}$ from a topological space $X$ to another topological space $Y$ is said to be a generalized Kakutani factorizable multifunction if there is a diagram

$$
T: X=X_{0} \stackrel{T_{0}}{\longrightarrow} X_{1} \stackrel{T_{1}}{\longrightarrow} \cdots \stackrel{T_{n}}{\longrightarrow} X_{n+1}=Y
$$

such that the following conditions are satisfied:

(1) $T_{0} \in \mathscr{K}\left(X, X_{1}\right)$;

(2) for each $i=1, \ldots, n+1$;

(a) $T_{i}$ is upper semicontinuous;

(b) for each $x \in T_{i-1}\left(X_{i-1}\right), T_{i}(x)$ is a nonempty subset of $X_{i}$;

(c) either $T_{i}$ is single-valued (in which case $X_{i+1}$ is required to be a Hausdorff topological vector space) or for each $x \in X_{i}, T_{i}(x)$ is a compact convex subset of $X_{i+1}$ (in which case $X_{i+1}$ is required to be a convex subset of a Hausdorff topological vector space).

The collection of all generalized Kakutani factorizable multifunctions is denoted by $\mathscr{K}_{C}^{\prime}(X, Y)$. 
The multifunctions $T_{i}$ are called factor multifunctions and the spaces $X_{i}$ are called factor spaces. It may be noted that if $T_{i}$ are multifunctions, then the factor spaces $X_{i}$ should be necessarily convex.

A similar definition for Kakutani factorizable multifunctions can be found in [6]. This version varies only in the nonempty conditions on the factor multifunctions where, as in [6], it is required that for each $x \in X_{i}, T_{i}(x)$ is a nonempty subset of $X_{i+1}$. Here the nonemptiness of the ultimate composition map only is assumed.

The proof of the following fixed-point theorem can be carried out in a similar fashion as in Lassonde [6].

THEOREM 3.1. If $S$ is a nonempty convex subset of a Hausdorfflocally convex topological vector space, then any compact generalized Kakutani factorizable multifunction $T: S \rightarrow 2^{S}$ (i.e., any compact multifunction in the family $\mathscr{K}_{C}^{\prime}(S, S)$ ) has a fixed point.

Let $A$ and $B$ be any two nonempty subsets of a normed linear space $E$. Let $B_{i}$ for $i=1, \ldots, n$ be nonempty subsets of $E$. Also let $A_{0}$, in this section, be the set $\left\{a \in A: d\left(a, b_{i}\right)=d\left(A, B_{i}\right)\right.$ for some $b_{i} \in B_{i}$ and for $\left.i=1, \ldots, n\right\}$.

The following best proximity theorem [19] is also utilized in the proof of Theorem 4.5.

THEOREM 3.2. Let $A$ be a nonempty compact convex subset and for each $i=1, \ldots, n$, let $B_{i}$ be a nonempty closed convex subset of a normed linear space $E$ such that $A_{0}$ is nonempty and compact. Further, let $T_{i}: A \rightarrow 2^{B_{i}}$ and let $i=1, \ldots, n$ be Kakutani multifunctions such that for each $x \in A_{0}$ and for each $y_{i} \in T_{i}(x)$, there exists $x_{0} \in A$ such that $\left\|x_{0}-y_{i}\right\|=d\left(A, B_{i}\right), i=1, \ldots, n$. Then there exists $x \in A_{0}$ such that

$$
d\left(x, T_{i}(x)\right)=d\left(A, B_{i}\right) \quad \text { for } i=1, \ldots, n .
$$

Sketch of the proof. Define $P: B=\prod_{i=1}^{n} B_{i} \rightarrow 2^{A_{0}}$ by

$$
P\left(\left(y_{1}, \ldots, y_{n}\right)\right)=\left\{x \in A:\left\|x-y_{i}\right\|=d\left(A, B_{i}\right) \forall i=1, \ldots, n\right\}
$$

Let $S: A_{0} \rightarrow 2^{A_{0}}$ be defined as $S=P \circ T$ where $T: A_{0} \rightarrow 2^{B}$ is defined by

$$
T(x)=\prod_{i=1}^{n} T_{i}(x) .
$$

It can be proved that $S$ is a compact generalized Kakutani factorizable multifunction [19]. 
Now, by invoking Theorem 3.1 to the multifunction $S$, there exists $x \in A_{0}$ such that $x \in S x$. So, $x \in P \circ T(x)$. This means that $x \in P(y)$ where $y=$ $\left(y_{1}, \ldots, y_{n}\right) \in T(x)$. By the definition of the multifunction $T$, it is clear that $y_{i} \in$ $T_{i}(x)$ for $i=1, \ldots, n$. Also, since $x \in P(y)$,

$$
\left\|x-y_{i}\right\|=d\left(A, B_{i}\right) \quad \text { for } i=1, \ldots, n \text {. }
$$

This completes the proof of the theorem

Example 3.3. Let $E=\mathbb{R}^{2}$ with the Euclidean norm.

Let

$$
\begin{aligned}
A & :=\{(x, 0): 0 \leq x \leq 3\}, \\
B_{1} & :=\{(x, y): y \geq 1 \text { and } 0 \leq x \leq 2\}, \\
B_{2} & :=\{(x, y): y \geq 1 \text { and } 1 \leq x \leq 3\} .
\end{aligned}
$$

Then

$$
A_{0}:=\{(x, 0): 1 \leq x \leq 2\}
$$

Let $T_{1}: A \rightarrow 2^{B_{1}}$ be defined as

$$
T_{1}(x, 0)= \begin{cases}\{(v, 1): v \in[0,1]\} & \text { if } x \neq 0, \\ \{(u, v): 0 \leq u \leq 2 v \geq 1\} & \text { otherwise }\end{cases}
$$

and $T_{2}: A \rightarrow 2^{B_{2}}$ be defined as

$$
T_{2}(x, 0)= \begin{cases}\{(v, 1): 1 \leq v \leq 3\} & \text { if } 0 \leq x \leq 1 \\ \{(x+1,1)\} & \text { otherwise. }\end{cases}
$$

It is easy to verify that all the conditions of Theorem 3.2 are satisfied and

$$
d\left((x, 0), T_{i}(x, 0)\right)=1=d\left(A, B_{i}\right) \quad \text { for } i=1,2, \forall 1 \leq x \leq 2 .
$$

The following best proximity pair theorem, due to Sadiq Basha and Veeramani [13], is a consequence of Theorem 3.2.

Corollary 3.4. Let $A$ be a nonempty, compact convex subset and let $B$ be a nonempty, closed and convex subset of a normed linear space $E$ such that $A_{0}$ is nonempty and compact. If $T: A \rightarrow 2^{B}$ is a Kakutani multifunction such that $T\left(A_{0}\right)$ $\subseteq B_{0}$, then there exists an element $x \in A$ such that $d(x, T(x))=d(A, B)$. 
Proof. Set $n=1$ in Theorem 3.2. In this case, it easy to observe that the condition $T\left(A_{0}\right) \subseteq B_{0}$ is equivalent to the condition in the hypothesis of the theorem, namely for each $x \in A_{0}$ and for each $y \in T(x)$ there exists $x_{0} \in A$ such that $\left\|x_{0}-y\right\|=d(A, B)$. Hence, $T$ satisfies all the conditions of Theorem 3.2 which proves the required result.

The next example exhibits the contrast between the best proximity pair theorems and the best approximation theorems

Example 3.5. Let $A=[0,1], B=[1,2]$, and $f: A \rightarrow B$ defined by $f(x)=x+$ 1. Clearly, $f$ is a continuous function, but there is no point $x \in A$ such that $d(x, T x)=d(A, B)$. This shows that the condition $f\left(A_{0}\right) \subseteq B_{0}$ is indispensable.

Also it is evident that $f$ satisfies all the conditions of the best proximity pair theorem and consequently 1 is the required best approximant.

This example, compared with Example 1.1, further illustrates the fact that the best proximity pair theorem aims at an approximate solution which is optimal.

Further, the contrast between the best proximity pair theorems and the best approximation theorems is that the best proximity pair theorem subsumes the fixed-point theorems for upper semicontinuous multifunctions whereas the best approximation theorems for multifunctions do not contain so because of the continuity assumption on the involved multifunctions.

Remark 3.6. In [13], Theorem 3.2 is proved in a more general setup where the set $A$ is approximately compact and $T$ is a Kakutani factorizable multifunction.

By choosing $B_{i}=A$ for each $i=1, \ldots, n$ in Theorem 3.2, the following common fixed-point theorem is obtained.

Corollary 3.7. Let $A$ be a nonempty convex subset of a normed linear space E. Suppose that $T_{i}: A \rightarrow 2^{A}, i=1, \ldots, n$, are Kakutani multifunctions such that for every $x \in A, \cap_{i=1}^{n} T_{i}(x) \neq \varnothing$, then there exists $x \in A$ such that $x \in T_{i}(x)$ for all $i=1, \ldots, n$.

Remark 3.8. It is remarked that the above corollary also follows from Lassonde's theorem [6] by observing that the map $F: A \rightarrow 2^{A}$, defined by $F(x)=\cap_{i=1}^{n} T_{i}(x)$, is a compact Kakutani self-multifunction and hence, by Lassonde's theorem, it has a fixed point.

\section{Applications to game theory}

The entire edifice of game theory expounds with a mathematical search to strike an optimal balance between persons generally having conflicting interests. Each player has to select one from his fixed range of strategies so as to bring the best outcome according to his own preferences. 
Following the pioneering work of Debreu [3], the generalized game is one in which the choices of each player is restricted to a subset of strategies determined by the choice of other players. Mathematically, the situation is described as follows: suppose there are $n$ players. Let $X_{i}$ be the strategy set and let $f_{i}: X=$ $\prod_{i=1}^{n} X_{i} \rightarrow \mathbb{R}$ be the pay-off function for the $i$ th player, for each $i=1, \ldots, n$. Given the strategies $x^{i}=\left(x_{1}, \ldots, x_{i-1}, x_{i+1}, \ldots, x_{n}\right)$ of all other players, the choice of the $i$ th player is restricted to the set $A_{i}\left(x^{i}\right) \subseteq X_{i}$. An equilibrium point in a generalized game is an element $x \in X$ such that for each $i=1, \ldots, n, x_{i} \in A_{i}\left(x^{i}\right)$ and

$$
\max _{y \in A_{i}\left(x^{i}\right)} f_{i}\left(y, x^{i}\right)=f_{i}\left(x_{i}, x^{i}\right)=f_{i}(x)
$$

where the following convenient notations are used.

Notation 4.1. Denote

$$
X=\prod_{i=1}^{n} X_{i}, \quad X^{i}=\prod_{\substack{j=1 \\ j \neq i}}^{n} X_{j} .
$$

It is written as $\left(x_{i}, x^{i}\right)$ for a point $x$ of $X$ for which its $i$ th coordinate is $x_{i}$ and $x^{i} \in X^{i}$.

The above definition of the equilibrium point is a natural extension of Nash equilibrium point introduced in [7]. Since then, a number of generalizations for the existence of an equilibrium point have been given in various directions. For instance, the existence results of equilibria of generalized games were given by Ding and Tan [4], Tan and Yuan [20], Tuclea [21], Lassonde [6], etc. For a unified treatment on the study of the existence of equilibria of generalized games in various settings, it is referred to Yuan [24].

Consider an economic situation where for each player two strategy sets $X_{i}$ and $Y_{i}$ are associated. The pay-off for each player is calculated by taking into account his choice of profitable strategy and independent strategy. So, let $f_{i}: \hat{Y}_{i} \times X \rightarrow \mathbb{R}$ be the pay-off for each player where

$$
\hat{Y}_{i}=Y_{i} \times \prod_{\substack{j=1 \\ j \neq i}}^{n} X_{j} .
$$

Also, let $A_{i}: X \rightarrow 2^{\widehat{Y_{i}}}$ be the constraint correspondence for each of the players. Moreover, the expenditure for each of the player on his travel from the two different strategy sets of him should also be taken into account. In this situation, we cannot expect an equilibrium point as the strategy sets $X_{i}$ and $Y_{i}$ may be quite different. Indeed, a main result of this section, Theorem 4.5 furnishes sufficient conditions to obtain a pair of $n$ points which behaves like an equilibrium point to the game and optimizes the travel expenditure for each of the players.

The following lemma is a key tool in the proof of Theorem 3.2. 
42 Best proximity pair theorems

Lemma 4.2. Let $A$ and $B$ be nonempty compact subsets of a normed linear space $F$ and $f: A \times B \rightarrow \mathbb{R}$ be a continuous function. Given a continuous multifunction $T$ : $A \rightarrow 2^{B}$ with compact values, the function $g: A \rightarrow \mathbb{R}$ defined by $g(x)=\delta(T x, x):=$ $\max _{z \in T(x)} f(z, x)$ is a continuous function.

Proof. Suppose $g$ is not continuous at some point $x_{0}$. Then, there exists an $\epsilon>0$ and a sequence $\left(x_{n}\right)$ such that $x_{n} \rightarrow x_{0}$

$$
\text { but }\left|g\left(x_{n}\right)-g\left(x_{0}\right)\right|>\epsilon \quad \text { for every } n \text {. }
$$

Now, choose $y_{0} \in T x_{0}$ such that

$$
g\left(x_{0}\right)=\delta\left(T x_{0}, x_{0}\right)=f\left(y_{0}, x_{0}\right)
$$

This choice is possible as $f$ is continuous and $T x_{0}$ is compact. Since $T$ is a lower semicontinuous multifunction by Theorem 2.2, there exist sequences $\left(x_{n_{k}}\right)$ and $\left(y_{n_{k}}\right)$ such that $y_{n_{k}} \in T x_{n_{k}}$ and $y_{n_{k}} \rightarrow y_{0}$. But,

$$
g\left(x_{0}\right)=f\left(y_{0}, x_{0}\right) \leq\left|f\left(y_{0}, x_{0}\right)-f\left(y_{n_{k}}, x_{n_{k}}\right)\right|+f\left(y_{n_{k}}, x_{n_{k}}\right) .
$$

As $y_{n_{k}} \in T x_{n_{k}}, f\left(y_{n_{k}}, x_{n_{k}}\right) \leq \delta\left(T x_{n_{k}}, x_{n_{k}}\right)=g\left(x_{n_{k}}\right)$. Therefore,

$$
g\left(x_{0}\right) \leq\left|f\left(y_{0}, x_{0}\right)-f\left(y_{n_{k}}, x_{n_{k}}\right)\right|+g\left(x_{n_{k}}\right) .
$$

Since $f$ is a continuous function, there exists an $m_{1} \in N$ such that for all $k>m_{1}$,

$$
\left(g\left(x_{0}\right)-g\left(x_{n_{k}}\right)\right) \leq \epsilon .
$$

Choose, for every $n, z_{n} \in T x_{n}$ such that

$$
g\left(x_{n}\right)=\delta\left(T x_{n}, y_{n}\right)=f\left(z_{n}, x_{n}\right)
$$

But, the sequence $\left(z_{n}\right) \subseteq Y$. As $Y$ is compact, the sequence $\left(z_{n}\right)$ has a convergent sequence $\left(z_{n_{k}}\right)$. Let $z_{n_{k}} \rightarrow z_{0}$. The upper semi-continuity of the multifunction $T$ implies that $z_{0} \in T x_{0}$ (by Theorem 2.1). Now,

$$
\begin{aligned}
g\left(x_{n_{k}}\right)= & f\left(z_{n_{k}}, x_{n_{k}}\right) \\
\leq & \left|f\left(z_{n_{k}}, x_{n_{k}}\right)-f\left(z_{0}, x_{0}\right)\right|+f\left(z_{0}, x_{0}\right) \\
\leq & \left|f\left(z_{n_{k}}, x_{n_{k}}\right)-f\left(z_{0}, x_{0}\right)\right|+g\left(x_{0}\right) \\
& \left(\text { since } z_{0} \in T x_{0}, f\left(z_{0}, x_{0}\right) \leq \delta\left(T x_{0}, x_{0}\right)=g\left(x_{0}\right)\right) .
\end{aligned}
$$


Again, as $f$ is a continuous function, there exists an $m_{2} \in N$ such that for all $k>m_{2}$,

$$
\left(g\left(x_{n_{k}}\right)-g\left(x_{0}\right)\right) \leq \epsilon .
$$

Choosing $m=\max \left\{m_{1}, m_{2}\right\}$, (4.8) and (4.11) imply, for all $k \geq m$,

$$
\left|g\left(x_{n_{k}}\right)-g\left(x_{0}\right)\right| \leq \epsilon
$$

eventually contradicting (4.4).

Let $X_{1}, \ldots, X_{n}$ and $Y_{1}, \ldots, Y_{n}$ be nonempty compact convex sets in a normed linear space $F$. Also, let $X=\prod_{i=i}^{n} X_{i}, Y=\prod_{i=1}^{n} Y_{i}$, and

$$
X_{0}=\{x \in X:\|x-y\|=d(X, Y) \text { for some } y \in Y\}
$$

Definition 4.3. Let $X$ be a normed linear space. A single-valued function $f: X \rightarrow$ $\mathbb{R}$ is said to be quasi-concave if the set

$$
\{x \in X: f(x) \geq t\}
$$

is convex for each $t \in \mathbb{R}$.

Let $X_{1}, \ldots, X_{n}$ and $Y_{1}, \ldots, Y_{n}$ be nonempty compact convex sets in a normed linear space $F$. Also, let

$$
X_{0}=\left\{x \in X:\left\|x-\hat{y}_{i}\right\|=d\left(X, \hat{Y}_{i}\right) \text { for some } \hat{y}_{i} \in \hat{Y}_{i}\right\}
$$

Definition 4.4. Let $f_{i}: \hat{Y}_{i} \times X \rightarrow \mathbb{R}$ for $i=1, \ldots, n$ be $n$ single-valued continuous functions. These $n$ functions are said to satisfy condition (B) with respect to the given compact-valued multifunctions $A_{i}: X \rightarrow 2^{\widehat{Y}_{i}}$ if for each $x \in X_{0}$ and for each $\hat{y}_{i} \in \hat{Y}_{i}$ such that

$$
\begin{gathered}
\hat{y}_{i} \in A_{i}(x), \\
\delta_{i}\left(A_{i}(x), x\right):=\max _{\hat{z} \in A_{i}(x)} f_{i}(\hat{z}, x)=f_{i}\left(\hat{y}_{i}, x\right), \quad i=1, \ldots, n,
\end{gathered}
$$

there exists $a \in X$ such that $\left\|a-\hat{y}_{i}\right\| \leq d\left(X, \hat{Y}_{i}\right)$.

TheOREM 4.5. Let $X_{1}, \ldots, X_{n}$ and $Y_{1}, \ldots, Y_{n}$ be nonempty compact convex sets in a normed linear space $F$. For $i=1, \ldots, n$, let $\hat{Y}_{i}=\prod_{j=1, j \neq i}^{n} X_{j} \times Y_{i}$; also let $f_{i}: \hat{Y}_{i} \times$ $X \rightarrow \mathbb{R}$ be continuous functions satisfying condition $(B)$ with respect to the given 
lower semicontinuous multifunctions $A_{i}: X \rightarrow 2^{\widehat{Y}_{i}}, i=1, \ldots, n$ in $\mathscr{K}\left(X, \widehat{Y}_{i}\right)$ and are such that for any fixed $x \in X_{i}$, the function $\hat{y}_{i} \rightarrow f_{i}\left(\hat{y}_{i}, x\right)$ is quasi-concave on $\hat{Y}_{i}$ for each $i=1, \ldots, n$. Then, there exist $x=\left(x_{1}, \ldots, x_{n}\right) \in X$ and $\hat{y}_{i}=\left(x_{1}^{\prime}, \ldots, x_{i-1}^{\prime}, y_{i}\right.$, $\left.x_{i+1}^{\prime}, \ldots, x_{n}^{\prime}\right) \in \hat{Y}_{i}, i=1, \ldots, n$ such that for each $i=1, \ldots, n$,

$$
\begin{gathered}
\hat{y}_{i} \in A_{i}(x) \\
\delta_{i}\left(A_{i}(x), x\right):=\max _{\hat{z} \in A_{i}(x)} f_{i}(\hat{z}, x)=f_{i}\left(\hat{y}_{i}, x\right) \\
\left\|x-\hat{y}_{i}\right\|=d\left(X, \hat{Y}_{i}\right), \quad\left\|x_{i}-y_{i}\right\|=d\left(X_{i}, Y_{i}\right) .
\end{gathered}
$$

Proof. For $i=1, \ldots, n$, let the multifunction $E_{i}: X \rightarrow 2^{\widehat{Y_{i}}}$ be defined as follows:

$$
E_{i}(x)=\left\{\hat{y}_{i} \in A_{i}(x): f_{i}\left(\hat{y}_{i}, x\right)=\delta_{i}\left(A_{i}(x), x\right)\right\}
$$

It is shown that $E_{i}$ satisfy all the conditions of Theorem 3.2. For this it is claimed that $E_{i} \in \mathscr{K}\left(X, \hat{Y}_{i}\right)$, for $i=1, \ldots, n$.

Let $i \in\{1, \ldots, n\}$ be fixed. For any fixed $x \in X, E_{i}(x)$ is nonempty and compact because the function $\hat{y}_{i} \rightarrow f_{i}\left(\hat{y}_{i}, x\right)$ is continuous on the compact set $A_{i}(x)$. Now, it is shown that $E_{i}(x)$ is convex

Let $z_{1}, z_{2} \in E_{i}(x)$. This implies

$$
\begin{aligned}
& f_{i}\left(z_{1}, x\right) \geq \delta_{i}\left(A_{i}(x), x\right), \\
& f_{i}\left(z_{2}, x\right) \geq \delta_{i}\left(A_{i}(x), x\right) .
\end{aligned}
$$

Since $\hat{y}_{i} \rightarrow f_{i}\left(\hat{y}_{i}, x\right)$ is quasi-concave on $\hat{Y}_{i}$,

$$
f_{i}\left(\lambda z_{1}+(1-\lambda) z_{2}, x\right) \geq \delta_{i}\left(A_{i}(x), x\right) .
$$

But, $A_{i}(x)$ is a convex set. So,

$$
f_{i}\left(\lambda z_{1}+(1-\lambda) z_{2}, x\right) \leq \delta_{i}\left(A_{i}(x), x\right) .
$$

Therefore,

$$
f_{i}\left(\lambda z_{1}+(1-\lambda) z_{2}, x\right)=\delta_{i}\left(A_{i}(x), x\right)
$$

Hence $\lambda z_{1}+(1-\lambda) z_{2} \in E_{i}(x)$. Therefore, $E_{i}(x)$ is convex for $i=1, \ldots, n$.

Next, it is shown that $E_{i}: X \rightarrow 2^{\widehat{Y}_{i}}$ is an upper semicontinuous multifunction on $X_{i}$. Let $z_{n} \in X$ with $z_{n} \rightarrow z$ and $w_{n} \in E_{i}\left(z_{n}\right)$ with $w_{n} \rightarrow w$.

The fact $w_{n} \in E_{i}\left(z_{n}\right)$ implies the fact that $f_{i}\left(w_{n}, z_{n}\right)=\delta_{i}\left(A_{i}\left(z_{n}\right), z_{n}\right)$. It follows that, by Lemma $4.2, x \rightarrow \delta_{i}\left(A_{i}(x), x\right)$ is a continuous function. So, $\delta_{i}\left(A_{i}\left(z_{n}\right), z_{n}\right) \rightarrow$ $\delta_{i}\left(A_{i}(z), z\right)$. Moreover, since $f_{i}$ is a continuous function $f_{i}\left(w_{n}, z_{n}\right) \rightarrow f_{i}(w, z)$. This 
implies that $f_{i}(w, z)=\delta_{i}\left(A_{i}(z), z\right)$. Hence $w \in E_{i}(z)$. Therefore, $E_{i}$ is upper semicontinuous on $X$ for every $i=1, \ldots, n$. Hence, this establishes the claim that $E_{i} \in \mathscr{K}\left(X, \hat{Y}_{i}\right)$, for $i=1, \ldots, n$.

Now, let $x \in X_{0}$ and $\hat{y}_{i} \in E(x)$. This implies that $f_{i}\left(\hat{y}_{i}, x\right)=\delta\left(A_{i}(x), x\right), i=$ $1, \ldots, n$. Since $f_{i}, i=1, \ldots, n$, satisfy condition (B) with respect to the multifunctions $A_{i}$, there exists $a \in X$ such that $\left\|a-\hat{y}_{i}\right\|=d\left(X, \hat{Y}_{i}\right)$. Hence, $E_{i}$ satisfy all the conditions of Theorem 3.2. Therefore, there exists $x \in X$ such that

$$
d\left(x, E_{i} x\right)=d\left(X, \hat{Y}_{i}\right)
$$

Since $E_{i} x$ is compact, there exists $\hat{y}_{i} \in E_{i} x$ such that

$$
\left\|x-\hat{y}_{i}\right\|=d\left(X, \hat{Y}_{i}\right) \quad \text { for } i=1, \ldots, n .
$$

Equipping the spaces $X$ and $\hat{Y}_{i}$ with product norm, the following result can be inferred:

$$
\begin{aligned}
d\left(X, \hat{Y}_{i}\right)= & \inf \left\{\left\|x_{1}-x_{1}^{\prime}\right\|+\cdots+\left\|x_{i}-y_{i}\right\|+\cdots+\left\|x_{n}-x_{n}^{\prime}\right\|:\left(x_{1}, \ldots, x_{n}\right) \in X\right. \\
& \left.\quad \text { and }\left(x_{1}^{\prime}, \ldots, y_{i}, \ldots, x_{n}^{\prime}\right) \in \hat{Y}_{i}\right\} \\
\leq & \inf \left\{\left\|x_{i}-y_{i}\right\|: x_{i} \in X_{i} \text { and } y_{i} \in Y_{i}\right\} \\
= & d\left(X_{i}, Y_{i}\right) .
\end{aligned}
$$

So,

$$
d\left(X_{i}, Y_{i}\right) \leq\left\|x_{i}-y_{i}\right\| \leq\left\|x-\hat{y}_{i}\right\|=d\left(X, \hat{Y}_{i}\right) \leq d\left(X_{i}, Y_{i}\right)
$$

Hence, $\left\|x_{i}-y_{i}\right\|=d\left(X_{i}, Y_{i}\right)$. This establishes the theorem.

Corollary 4.6. Let $X_{1}, \ldots, X_{n}$ be nonempty compact convex sets in a normed linear space $F$. Also, let $f_{i}: X \times X \rightarrow \mathbb{R}$ be continuous functions, $X=\prod_{i=1}^{n} X_{i}$, such that for any fixed $x \in X$, the function $y \rightarrow f_{i}(y, x)$ is quasi-concave on $X$ for each $i=1, \ldots, n$. Then, there exists $x \in X$ such that for each $i=1, \ldots, n$,

$$
\max _{z \in X} f_{i}(z, x)=f_{i}(x, x) .
$$

Proof. Choose $Y_{i}=X_{i}$ and $A_{i}: X \rightarrow 2^{X}$ as $A_{i}(x)=X$ for all $i=1, \ldots, n$. As the single-valued continuous functions satisfy condition (B) automatically, the above theorem ensures the existence of $x \in X$ and $\hat{y}_{i} \in X, i=1, \ldots, n$, such that for each $i=1, \ldots, n$,

$$
\begin{gathered}
\max _{\hat{z} \in X} f_{i}(\hat{z}, x)=f_{i}\left(\hat{y}_{i}, x\right), \\
\left\|x-\hat{y}_{i}\right\|=d(X, X)=0, \quad\left\|x_{i}-y_{i}\right\|=d\left(X_{i}, X_{i}\right)=0 .
\end{gathered}
$$

Therefore, $\hat{y}_{i}=x$ for all $i=1, \ldots, n$ and this completes the proof. 
A particular case of the above corollary is the following.

Corollary 4.7. Let $X$ be a nonempty compact convex subset of a normed linear space $F$. Let $f: X \times X \rightarrow \mathbb{R}$ be a continuous function such that for each fixed $x \in X$, the function $y \rightarrow f(y, x)$ is quasi-concave. Then, there exists a point $x \in X$ such that

$$
\max _{z \in X} f(z, x)=f(x, x)
$$

Remark 4.8. In the above corollary, and hence in the main theorem, the quasiconcavity condition on $f$ cannot be dropped.

Let $X$ be any infinite compact convex subset of a normed linear space $F$. Let $f: X \times X \rightarrow \mathbb{R}$ be defined as $f((x, y))=\|x-y\|$. Then, there cannot exist any $x \in X$ such that

$$
\max _{z \in X} f(z, x)=f(x, x)
$$

\section{Acknowledgments}

The authors thank the referee for his valuable suggestions for the improvement of the paper. The second author would like to thank Prof. Dan Butnariu, University of Haifa, Israel and Prof. Simeon Reich, The Technion-Israel Institute of Technology, for the fruitful discussions he had with them and also for the local hospitality extended by them during his short visits to their departments. This paper is based on the talk given by the second author at the International Conference on Fixed-Point Theory and Its Applications held at the Technion-Israel Institute of Technology, Haifa, Israel during 13-19 June, 2001.

\section{References}

[1] J.-P. Aubin and I. Ekeland, Applied Nonlinear Analysis, John Wiley \& Sons, New York, 1984.

[2] G. Beer and D. Pai, Proximal maps, prox maps and coincidence points, Numer. Funct. Anal. Optim. 11 (1990), no. 5-6, 429-448.

[3] G. Debreu, A social equilibrium existence theorem, Proc. Nat. Acad. Sci. U.S.A. 38 (1952), 886-893.

[4] X. P. Ding and K.-K. Tan, On equilibria of noncompact generalized games, J. Math. Anal. Appl. 177 (1993), no. 1, 226-238.

[5] K. Fan, Extensions of two fixed point theorems of F. E. Browder, Math. Z. 112 (1969), 234-240.

[6] M. Lassonde, Fixed points for Kakutani factorizable multifunctions, J. Math. Anal. Appl. 152 (1990), no. 1, 46-60.

[7] J. Nash, Equillibrium points in N-person games, Proc. Nat. Acad. Sci. U.S.A. 36 (1950), 48-49.

[8] D. V. Pai, Proximal points of convex sets in normed linear spaces, Yokohama Math. J. 22 (1974), 53-78. 
[9] A A characterization of smooth normed linear spaces, J. Approximation Theory 17 (1976), no. 4, 315-320.

[10] J. B. Prolla, Fixed-point theorems for set-valued mappings and existence of best approximants, Numer. Funct. Anal. Optim. 5 (1982/83), no. 4, 449-455.

[11] S. Reich, Fixed points in locally convex spaces, Math. Z. 125 (1972), 17-31.

[12] - Approximate selections, best approximations, fixed points, and invariant sets, J. Math. Anal. Appl. 62 (1978), no. 1, 104-113.

[13] S. Sadiq Basha and P. Veeramani, Best proximity pairs and best approximations, Acta Sci. Math. (Szeged) 63 (1997), no. 1-2, 289-300.

[14] Best proximity pair theorems for multifunctions with open fibres, J. Approx. Theory 103 (2000), no. 1, 119-129.

[15] B. N. Sahney and S. P. Singh, On best simultaneous approximation, Approximation Theory, III (Proc. Conf., Univ. Texas, Austin, Tex., 1980), Academic Press, New York, 1980, pp. 783-789.

[16] V. M. Sehgal and S. P. Singh, A generalization to multifunctions of Fan's best approximation theorem, Proc. Amer. Math. Soc. 102 (1988), no. 3, 534-537.

[17] A theorem on best approximations, Numer. Funct. Anal. Optim. 10 (1989), no. 1-2, 181-184.

[18] I. Singer, Best Approximation in Normed Linear Spaces by Elements of Linear Subspaces, Publishing House of the Academy of the Socialist Republic of Romania, Bucharest, 1970.

[19] P. S. Srinivasan and P. Veeramani, Best proximity pair theorems for a family of sets, Analysis and Its Applications (Chennai, 2000), Allied Publ., New Delhi, 2001, pp. 197-207.

[20] K.-K. Tan and X.-Z. Yuan, Approximation method and equilibria of abstract economies, Proc. Amer. Math. Soc. 122 (1994), no. 2, 503-510.

[21] C. I. Tulcea, On the approximation of upper semi-continuous correspondences and the equilibriums of generalized games, J. Math. Anal. Appl. 136 (1988), no. 1, 267289.

[22] V. Vetrivel, P. Veeramani, and P. Bhattacharyya, Some extensions of Fan's best approximation theorem, Numer. Funct. Anal. Optim. 13 (1992), no. 3-4, 397-402.

[23] X. B. Xu, A result on best proximity pair of two sets, J. Approx. Theory 54 (1988), no. 3, 322-325.

[24] G. X.-Z. Yuan, The study of minimax inequalities and applications to economies and variational inequalities, Mem. Amer. Math. Soc. 132 (1998), no. 625, x+140.

P. S. Srinivasan: Department of Mathematics, Indian Institute of Technology (IITM), Madras, Chennai 600 036, India

P. Veeramani: Department of Mathematics, Indian Institute of Technology (IITM), Madras, Chennai 600 036, India

E-mail address: pvmani@iitm.ac.in 


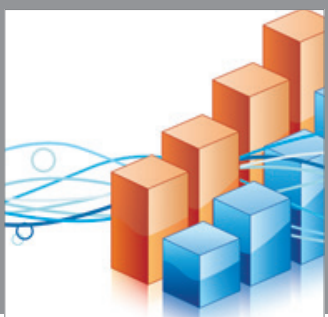

Advances in

Operations Research

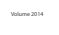

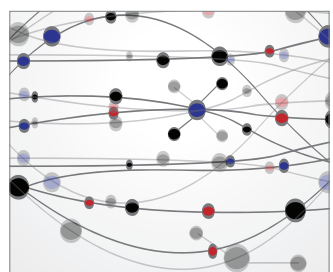

\section{The Scientific} World Journal
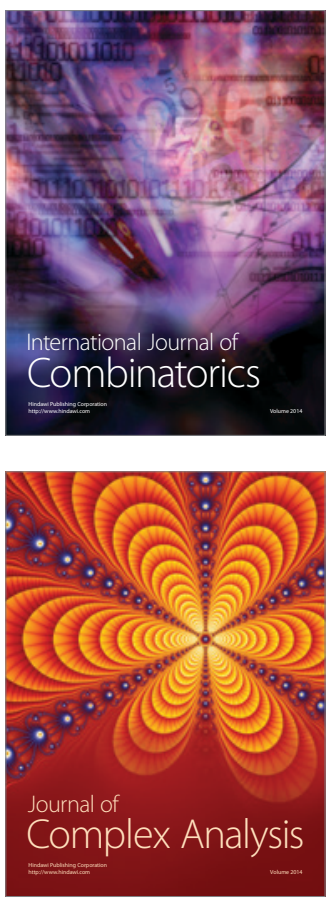

International Journal of

Mathematics and

Mathematical

Sciences
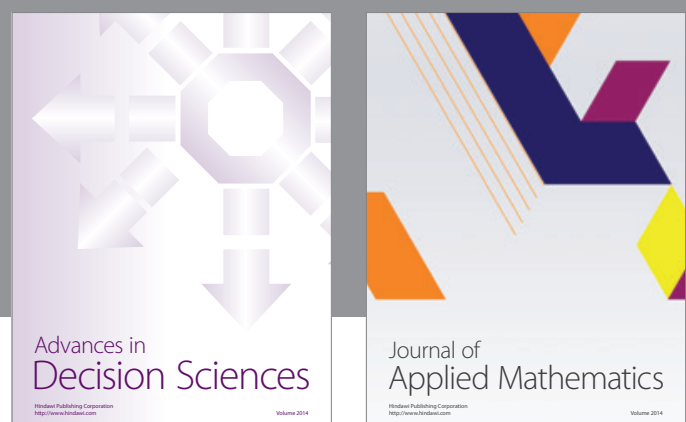

Journal of

Applied Mathematics
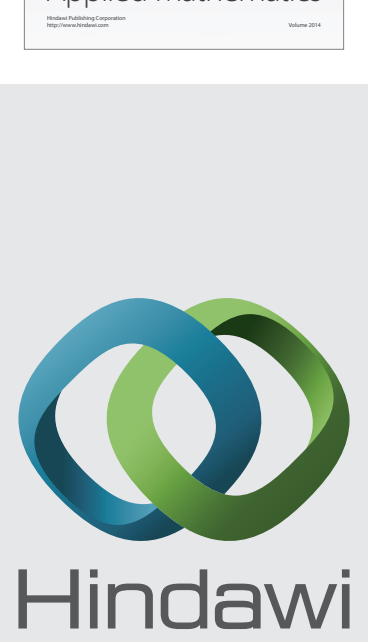

Submit your manuscripts at http://www.hindawi.com
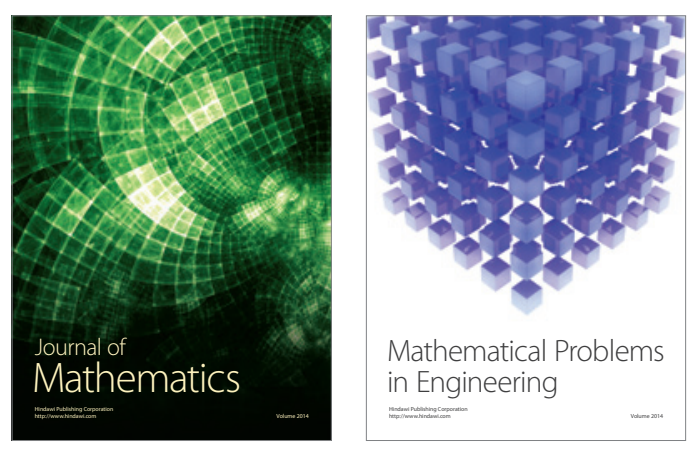

Mathematical Problems in Engineering
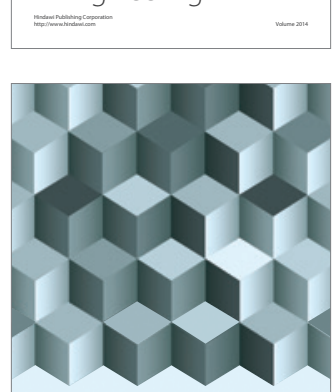

Journal of

Function Spaces
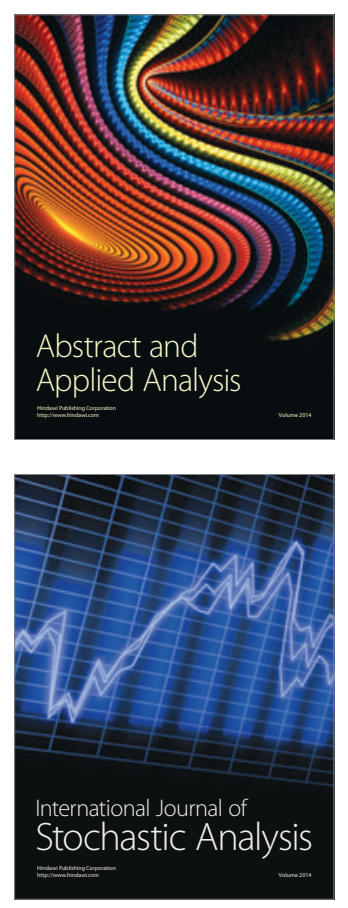

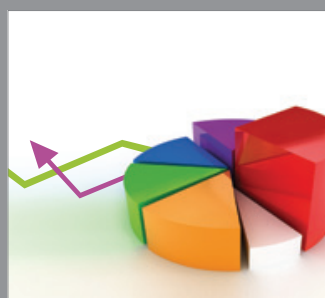

ournal of

Probability and Statistics

Promensencen
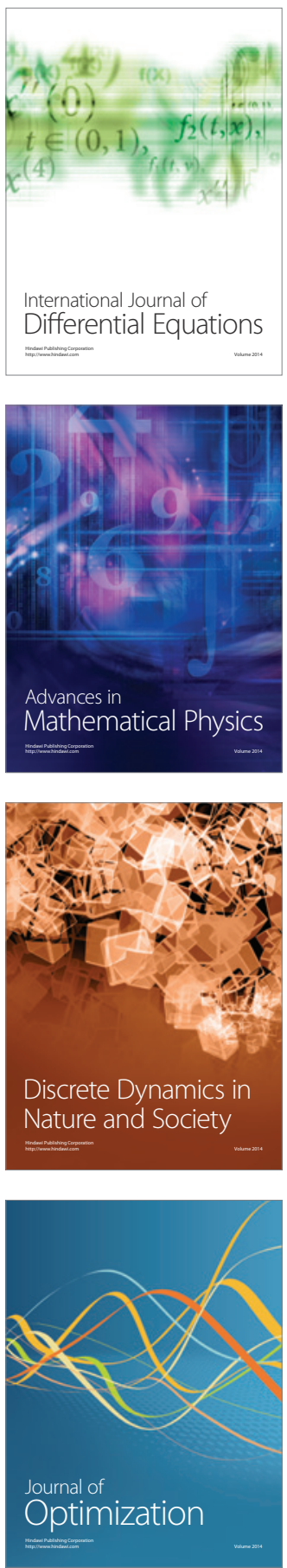